\title{
Immunotoxicity and allergenic properties of humic acids isolated from peloid
}

Zhernov Yu.V. ${ }^{1}$, Litovkina A.O. ${ }^{1}$, Perminova I.V. ${ }^{2}$, Khaitov M.R. $^{1}$

${ }^{1}$ National Research Center - Institute of Immunology FMBA of Russia, Moscow, Russia, y.zhernov@nrcii.ru

${ }^{2}$ Lomonosov MSU, Department of Chemistry, Moscow, Russia

doi: 10.36291/HIT.2019.zhernov.018

Currently, biologically active food additives (BAFA) based on humic acids (HA) are widely distributed, which leads to the appearance of clinical cases associated with development of atypical reactions after ingestion of these supplements. HA are known to possess a broad spectrum of pharmaceutical activity, including pronounced antiinflammatory and immunomodulatory properties. This fact stimulated us to study a complex evaluation of the immunotoxicity and allergenic properties of $\mathrm{HA}$.

The low-mineralized silt sulfide mud (peloid) samples were collected during spring, 2017 from the Molochka Lake of the Samara region, Russia. HA were isolated from peloid samples using extraction with $0.5 \mathrm{M} \mathrm{NaOH}$ as described in [1]. The immunotoxicity and allergenic properties of HA were evaluated according to the methodological recommendations of the European Medicines Agency and Research center for medical application, Russia. [2]. The effect of HA on the humoral immune response was evaluated by the change in the number of antibody-forming cells (AFC) in the spleen of mice when they were immunized with sheep red blood cells (SRBC) [3]. The effect of HA on the cellmediated immune response was evaluated on the model of induction of the type IV hypersensitivity reaction to hapten reagent 2,4,6-trinitrobenzenesulfonic acid (TNBS) (Sigma-Aldrich) [4]. The effect of HA on neutrophil activation was evaluated by phorbol 12myristate 13-acetate (PMA) and zymosan was assessed by luminol-dependent chemiluminescence $(C L)$ [5]. The serum total IgE level after intraperitoneal administration of $\mathrm{HA}$ to BALB/c mice was studied by the solid-phase ELISA.

HA extract was studied in complex tests on a female F1 (CBA x C57BL 6) hybrid mice and BALB/c mice weighing 18-24 g six weeks of age. Administered per orally in doses of 100 and $1000 \mathrm{mg} / \mathrm{kg}$ (p.o.), HA exhibited neither immunotoxic nor allergenic effects. In CL tests, HA administered in mice in a dose of $100 \mathrm{mg} / \mathrm{kg}$ (p.o.) during 14 days inhibited the production of reactive oxygen species by neutrophils. HA administered to BALB/c mice in doses of 100 and $1000 \mathrm{mg} / \mathrm{kg}$ reduced the intensity of systemic anaphylaxis.

In conclusion, the results of our experiments indicate that HA from peloids does not exhibit undesired immunotoxicity and allergenic properties and however pose questions for the further investigation of BAFA based on HA as a potential allergenic agent.

References

1. Agapov A.I., Avvakumova N.P. // Patent RU 2028149, July 20, 1999.

2. Neuman D.A. // Fd Chem Toxic. 1995. 33(10):887-894.

3. Ladics G.S. // Methods. 2007. 41(1):9-19.

4. Wirtz S. et al. // Nat Protoc. 2017. 12(7):1295-1309.

5. Lieberman M.M. et al. // Clin Diagn Lab Immunol. 1996. 3(6):654-662. 